\title{
A contribuição da pesquisa para convergência e crescimento da renda agropecuária no Brasil ${ }^{1}$
}

\author{
Ricardo Candéa Sá Barreto² \\ Eduardo Almeida ${ }^{3}$
}

\begin{abstract}
Resumo: $\mathrm{O}$ setor agropecuário no Brasil passou por diversas mudanças nas últimas décadas, tanto na composição das políticas públicas voltadas ao setor, quanto no aspecto tecnológico. Este artigo estuda a influência dos investimentos em pesquisa agropecuária para o crescimento econômico e a convergência de renda agropecuária no período de 1986 a 2004. Para isso, utiliza-se um modelo econométrico de efeitos fixos, controlando-se os efeitos espaciais, ou seja, a dependência espacial e a heterogeneidade espacial. Além de comprovar convergência condicional, o modelo revelou que o crescimento do PIB per capita agropecuário foi afetado por variáveis como capital físico agropecuário, infra-estrutura telefônica, infra-estrutura de armazenagem, crédito rural, número de trabalhadores rurais e externalidades espaciais. Além disso, ficou evidenciada a importância de P\&D agropecuário para o crescimento e convergência de renda per capita no setor agropecuário.
\end{abstract}

Palavras-chaves: convergência de renda, agropecuária, modelo de efeitos fixos com dependência espacial.

Abstract: Over the last decades, the Brazilian agricultural and livestock sector has presented several changes, both in terms of public policies and of the technological aspect. This article examines the economic growth, as well as the convergence of farmer's income Brazilian states, from 1986 to 2004. An econometric model of fixed effects is used, controlling spatial effects. In addition to proving conditional convergence, the econometric model revealed that the growth in per capita GDP for the agricultural and livestock sector was affected by variables such as agricultural physical capital, telecommunication

${ }^{1}$ Eduardo Almeida agradece o apoio do Conselho Nacional de Desenvolvimento Científico e Tecnológico (CNPq), Fundação de Amparo à Pesquisa do Estado de Minas Gerais (Fapemig) e à Universidade Federal de Juiz de Fora para a realização deste artigo.

${ }^{2}$ Professor Doutor do programa de mestrado em economia aplicada da Universidade Federal de Juiz de Fora. E-mail: ricardo.candea@ufjf.edu.br

${ }^{3}$ Professor Adjunto da Universidade Federal de Juiz de Fora e pesquisador do CNPq. E-mail: eduardo.almeida@ufjf.edu.br 
infrastructure, storage infrastructure, rural credit, rural workers and spatial externalities. Furthermore, this paper highlights the importance of $R \mathcal{E}$ D investments for growth and convergence of per capita income in agricultural and livestock sector.

Key-words: income convergence, agricultural and livestock sector, fixed effect model with spatial dependence.

Classificação JEL: O18, O47, R11, R23.

\section{Introdução}

O setor agropecuário no Brasil passou por diversas mudanças nas últimas décadas, tanto na composição dos gastos do governo voltados ao setor quanto no aspecto tecnológico. Isso, por sua vez, modificou o seu padrão de produtividade, principalmente após o início da década de 1990.

Sob uma perspectiva histórica, a transformação da agricultura brasileira acontece a partir de meados da década de 60, quando se insere no contexto da modernização e desenvolvimento do País, introduzido pelo Governo de Juscelino Kubitschek. Inicialmente, foram nas regiões do Sul e Sudeste do Brasil que a agricultura se desenvolveu de forma intensiva. Entretanto, devido ao esgotamento de terras disponíveis para a ocupação da agropecuária e à necessidade de aumento da produtividade agrícola, houve o direcionamento da produção para novas áreas e a conseqüente expansão agrícola.

Tendo-se em mente a grande importância dos investimentos em pesquisa, $\mathrm{o}$ marco fundamental das novas políticas é a criação da Empresa Brasileira de Pesquisa Agropecuária (Embrapa) em 1973. Conforme Bonelli e Pessoa (1998), as despesas em pesquisa efetuadas por essa empresa aumentaram rapidamente nos anos 70, tanto em termos reais quanto em relação ao PIB agrícola, mas permaneceram praticamente constantes até o início dos anos $90^{4}$. As pesquisas da Embrapa produziram importantes inovações nas áreas de tecnologias bioquímicas, técnicas de manejo do solo e adaptações no cultivo para as condições agroecológicas prevalecentes no Brasil — a bem-sucedida adaptação das variedades de soja ao solo do Cerrado ratifica essa visão. ${ }^{5}$

\footnotetext{
${ }^{4}$ Segundo Beintema et al. (2001), grande parte da pesquisa é desenvolvida por organizações públicas, sendo que $79 \%$ deste total por organizações federais e estaduais e $15 \%$ por agências de ensino superior. A Embrapa ainda é a organização mais importante de pesquisa do País, sendo responsável por mais de $57 \%$ dos investimentos feitos em pesquisa agropecuária.

${ }^{5}$ A modernização foi, sem dúvida, grandemente estimulada pela atuação governamental em diversos níveis. A política agrícola beneficiou-se especialmente de: $a$ ) mudanças na estrutura de incentivos implícita nos instrumentos de política macroeconômica, destacando-se as políticas de crédito e de preços mínimos e as desvalorizações cambiais; b) maciços investimentos na infra-estrutura de pesquisa; c) expansão e integração do sistema viário nacional. Para mais detalhes, ver Bonelli e Pessoa (1998).
} 
É possível que os investimentos em pesquisa tenham contribuído para a convergência de renda agrícola no Brasil, visto que as inovações originadas na Embrapa tiveram, no entanto, tecnologias poupadoras de terra que aumentaram o nível de renda rural, ao mesmo tempo contribuíram para incorporação de novas áreas no Centro-Oeste, sul dos estados do Pará, Piauí e Maranhão, além do oeste da Bahia e estados como Rondônia e Acre. Como essas áreas também se adequavam à mecanização, conclui-se que essas inovações revestiam-se, ainda, de inovações tecnológicas poupadoras de mão-de-obra.

Objetiva-se, neste artigo, avaliar se os investimentos em pesquisa agropecuária contribuíram para a diminuição das desigualdades do PIB por trabalhador agropecuário e/ou contribuíram para o crescimento do PIB por trabalhador agropecuário nos estados brasileiros ao longo do período de 1985 a 2004, além de se comprovar se ocorreu convergência de renda agropecuária no período.

Para isso, a metodologia a ser utilizada neste trabalho consta de um modelo econométrico de efeitos fixos com controle para a dependência espacial ${ }^{6}$. Esse modelo procura controlar tanto a dependência espacial quanto a heterogeneidade espacial, representada por diferentes interceptos.

Os resultados indicaram que, no período de 1986 a 2004, houve uma tendência à equalização das rendas por trabalhador agropecuário entre os estados brasileiros. Dentro dessa perspectiva mais ampla de convergência condicional, os resultados mostraram, também, que a pesquisa agropecuária contribuiu tanto para o crescimento da renda do setor quanto para a sua convergência entre os estados brasileiros.

Além desta seção introdutória, este trabalho está estruturado como se segue. Na próxima seção, uma revisão da literatura. Em seqüência, apresenta-se o modelo empírico-econométrico. Na quarta parte, destacam-se as fontes de dados e as definições das variáveis importantes na obtenção do controle condicional do efeito de convergência. As evidências empíricas são apresentadas e discutidas na quinta seção. Expõem-se, na última seção, as conclusões de relevo e tecem-se as considerações finais.

\section{Revisão de literatura}

À luz das teorias do crescimento endógeno, o progresso tecnológico ocorre quando as empresas ou os inventores maximizadores de lucro buscam desenvolver novos e melhores produtos. É a possibilidade de auferir lucro que leva as empresas a desenvolverem um novo produto, processo ou idéia. Assim, as melhorias tecnológicas e o próprio processo de crescimento econômico são compreendidos como resultado endógeno da economia.

Robert Solow (1956), no chamado Modelo Neoclássico de Crescimento Econômico, aponta que o progresso tecnológico emana fora do contexto da

\footnotetext{
${ }^{6}$ Cabe destacar ainda, segundo Elhorst (2003) e Arbia (2006), que a combinação de dados de painel com modelos espaciais encontra-se na fronteira de pesquisa sobre o tema.
} 
economia (exógeno), enquanto as contribuições de Paul Romer (1986) e Robert Lucas (1988) incorporam os retornos crescentes de escala, e o progresso tecnológico pode ser pensado como uma outra forma de acumulação de capital a partir de empresas que desenvolvam Pesquisa e Desenvolvimento (P\&D).

Para Howitt e Mayer-Foulkes (2005), o nível geral de P\&D da economia determina o fluxo de inovações da economia, que, por seu turno, governa a taxa de progresso tecnológico, e, portanto, determina a taxa de crescimento econômico de longo prazo. Dado que as variáveis institucionais e políticas são mantidas constantes, um aumento no estoque de capital por unidade de trabalho irá elevar o incentivo a desenvolver P\&D por meio do efeito escala. Isto é, mais capital por trabalhador irá resultar em mais produção por trabalhador, e daí, mais renda per capita, para um dado nível de tecnologia. E quando as pessoas têm rendas maiores, estas podem gastar mais em produtos recentemente inventados, o que eleva o incentivo a desenvolver P\&D e resulta em uma taxa mais rápida de crescimento. Os autores ainda apontam que a introdução dos modernos P\&D foi a força fundamental por trás da emergência das grandes disparidades de renda.

No que tange a renda agropecuária, existe na literatura um conjunto de trabalhos que, usando da abordagem da convergência, procura estudar variáveis vinculadas à agricultura, tais como a renda por trabalhador, produção ou a produtividade. Nesse sentido, ressalta-se o trabalho de Lusigi e Thirtle (1998) para 32 países africanos entre 1970 e 1991, que comprovou a existência de convergência condicional da renda por trabalhador como da produtividade total dos fatores na agricultura, tendo como variáveis de controle adotadas: investimento, educação, participação na agricultura e emprego.

Já Gutierrez (2000) encontrou convergência absoluta da produtividade do trabalho agrícola nas unidades federativas dos Estados Unidos da América para o período de 1970 a 1992. A mesma análise para os países da União Européia não mostrou evidências de convergência absoluta da produtividade do trabalho agrícola. Resultado semelhante foi encontrado por Suhariyanto e Thirtle (2001). Analisando a produtividade agrícola na Ásia entre 1965 a 1996, os autores não encontraram evidências de convergência para a produtividade total dos fatores na agricultura.

No Brasil, Oliveira Junior et al. (2007) encontraram evidências de convergência condicional microrregional do valor da produção agrícola por trabalhador no período de 1970 a 1996, encontrando, também, evidências de que o capital físico é mais importante que o capital humano na explicação do processo de crescimento.

Almeida et al. (2008) investigaram se há convergência espacial da produtividade agrícola no período mais recente entre 1991 e 2003 para as microrregiões do Brasil. O estudo apresenta evidências em favor da existência de convergência absoluta da produtividade agrícola, porém, num ritmo muito lento no período de 1991 a 2003 e 1991 a 1994. Para os subperíodos 1995-1999 e 2000-2003, contudo, não se observa a convergência de renda.

O setor agropecuário é sujeito à influência de efeitos de vizinhança, efeitos de contágio (de pragas) e de inovação tecnológica. Nesse sentido, Anselin (1988) 
ressalta a importância da dependência espacial e afirma que tal efeito pode ser entendido como a falta de independência entre observações em uma cross section ou dados de painel. Em particular, como este artigo tem como o foco os investimentos de pesquisa da Embrapa, espera-se teoricamente verificar efeitos de spillover e de difusão tecnológica proveniente de inovações introduzidas pela Embrapa que ocorrem por meio das regiões. Em vista disso, por constituir num elemento importante, explicando o crescimento entre países, a dimensão geográfica deve ser estudada com muita atenção.

\section{Modelo ${ }^{7}$}

Utiliza-se como referência um modelo econométrico espacial de efeitos fixos com dependência espacial, como especificado abaixo na forma de dados empilhados: ${ }^{8}$

$G_{t}=\mu+\rho W_{1} G_{t}+\beta \ln Y_{t-1}+X_{t-1} \alpha+W_{1} X_{t-1} \tau+\phi P \& D_{t-1}+\gamma P \& D_{t-1} \ln Y_{t-1}+u_{t}$ $u_{t}=\lambda W_{2} u_{t}+\varepsilon_{t}$

em que $G_{t}$ é um vetor do logaritmo da relação do PIB por trabalhador do período corrente e o do PIB por trabalhador agropecuário do período anterior para os anos entre 1986 e 2004, ao passo que $W_{1} G_{t}$ é a variável dependente defasada espacialmente. $\mathrm{O}$ parâmetro $\rho$ é o coeficiente de defasagem espacial, que capta os efeitos de transbordamento do PIB por trabalhador sobre os vizinhos.

O vetor $\mu_{i}=\left(\mu_{1}, \ldots, \mu_{k}\right)$ representa os efeitos fixos. O modelo de efeitos fixos assume que as diferenças das unidades são captadas nos diferentes interceptos, mas têm as mesmas inclinações. Nesse sentido, os efeitos fixos são os efeitos específicos não-observáveis e constantes ao longo do tempo, atribuíveis a características específicas de cada região.

$\operatorname{Ln} Y_{t-1}$ é o nível da renda per capita no período inicial e o coeficiente $\beta$ fornece a informação sobre a convergência.

A matriz $X_{t-1}$ representa as variáveis de controle iniciais (de 1985 a 2003), ${ }^{9}$ sendo $\alpha$ o seu vetor de coeficientes a serem estimados. $W_{1}$ e $W_{2}$ são os operadores de defasagem espacial, $\varepsilon$ são os termos de erro i.i.d. $\operatorname{com} E(\varepsilon)=0 \mathrm{e} E\left(\varepsilon \varepsilon^{\prime}\right)=\sigma^{2} I_{N T}$.

Semelhantemente, constrói-se uma defasagem espacial das variáveis explicativas iniciais $W_{1} X_{t-1}$. Assim, o vetor de coeficientes $\tau$ representa as externalidades que cada variável de controle condicional de uma região exerce sobre outras, influenciando o crescimento econômico dos estados.

\footnotetext{
7 O modelo teórico é baseado na análise de convergência da renda. Para uma descrição da teoria subjacente, consulte Barro e Sala-i-Martin (1995).

${ }^{8}$ Para uma referência da notação para dados em painel empilhados, Elhorst (2003).

9 A utilização de variáveis defasadas em um ano tem o objetivo de minimizar problemas de endogeneidade. Para mais detalhes, ver Arellano e Bond (1991).
} 
Já o coeficiente $\phi$ quantifica a contribuição da pesquisa agropecuária $\left(P \& D_{t-1}\right)$ realizada no período anterior, para o crescimento da renda agropecuária por trabalhador e $\gamma$ quantifica a contribuição da $P \& D_{t-1}$ para a sua convergência.

O estimador de efeitos fixos permite controlar esses componentes e eliminar, em larga medida, o viés das variáveis omitidas. Portanto, a estimação do modelo espacial por efeitos fixos tem a vantagem de controlar este tipo de heterogeneidade, considerando, ainda, a dependência espacial dos dados. A heterogeneidade espacial ${ }^{10}$ diz respeito à falta de estabilidade de comportamento através do espaço, como, por exemplo, municípios ricos e pobres aglutinados em diferentes regiões de um país.

\section{Dados}

O painel de dados contém a totalidade dos 26 estados brasileiros e o Distrito Federal, no período de 1985 a 2004. Uma série de variáveis proxies é definida no Quadro ${ }^{11}$. Em seguida, é feita uma descrição das variáveis usadas para estimar os resultados.

A variável dependente $G_{t}$ é medida como o logaritmo natural do fator de crescimento:

$$
G_{t}=\ln \left(\frac{Y_{t}}{Y_{t-1}}\right)
$$

em que Yé o PIB por trabalhador.

Quanto à escolha das variáveis explicativas $X_{t-1}$ do modelo empírico, procura-se controlar diversas características regionais que atuam sobre $o$ desenvolvimento. Convém recordar que variáveis explicativas representam a matriz $X_{t-1}$ para cada estado, ou seja, as características iniciais (referentes ao ano inicial do período) de cada uma. A matriz $X_{t-1}$ é composta pelas seguintes variáveis:

1. O número de trabalhadores formais ( $\left.\mathrm{Tra}_{t-1}\right)$ nos setores agricultura, borracha, fumo, couro, madeira e mobiliário foi obtido no Cadastro Geral de Empregado e Desempregado (Caged), do Ministério do Trabalho e Emprego (MTE).

2. Densidade Rodoviária $\left(D R_{t-1}\right)$ denota a extensão das rodovias federais pavimentadas relativas à área de cada estado.

\footnotetext{
${ }^{10}$ Anselin (1988) trata a heterogeneidade espacial como uma forma de instabilidade dos parâmetros, isto é, a forma funcional e/ou os parâmetros do modelo podem mudar de acordo com as localizações geográficas ou variáveis no conjunto. Outra fonte de heterogeneidade espacial reside na variância não-constante.

${ }^{11}$ Nos anexos 1 e 2 podem ser verificadas as estruturas da distribuição das principais informações estatísticas utilizadas para o ano de 2004, como a variação percentual dessas informações no período de 1985 a 2003.
} 
3. Infra-estrutura de telecomunicação por trabalhador $\left(I T_{t-1}\right)$ tem como proxy os terminais telefônicos fixos totais do Brasil (residenciais e públicos), em serviço, relativos ao número de trabalhadores de cada estado.

4. A capacidade estática dos armazéns $\left(C O N A B_{t-1}\right)$ por estado da união, em mil toneladas, foi obtida na Companhia Nacional de Abastecimento (Conab).

Quadro 1. Variáveis consideradas para o crescimento econômico dos estados ${ }^{12}$.

\begin{tabular}{|c|c|c|c|c|c|}
\hline Variável & Descrição & $\begin{array}{c}\text { Sinal } \\
\text { esperado }\end{array}$ & $\begin{array}{c}\text { Referencial } \\
\text { teórico }\end{array}$ & $\begin{array}{c}\text { Referencial } \\
\text { empírico }\end{array}$ & Fonte \\
\hline$G_{t}$ & $\begin{array}{c}\text { Logaritmo } \\
\text { natural do fator } \\
\text { de crescimento }\end{array}$ & & Solow (1956) & $\begin{array}{l}\text { Oliveira Júnior; } \\
\text { Castelar; e } \\
\text { Ferreira (2007) }\end{array}$ & $\begin{array}{l}\text { IBGE (2007); } \\
\text { MTE (2007). }\end{array}$ \\
\hline $\ln \left(Y_{t-1}\right)$ & $\begin{array}{l}\text { Logaritmo } \\
\text { natural do PIB } \\
\text { por trabalhador } \\
\text { agropecuário } \\
\text { no início do } \\
\text { período } \\
\end{array}$ & - & Solow (1956) & $\begin{array}{l}\text { Oliveira Júnior; } \\
\text { Castelar; e } \\
\text { Ferreira (2007) }\end{array}$ & $\begin{array}{l}\text { IBGE (2007); } \\
\text { MTE (2007). }\end{array}$ \\
\hline$D R_{t-1}$ & $\begin{array}{l}\text { Densidade } \\
\text { Rodoviária }\end{array}$ & + & $\begin{array}{l}\text { Zhang e Fan } \\
\qquad(2004)\end{array}$ & Barreto (2007) & $\begin{array}{l}\text { IPEA (2007); } \\
\text { DNIT (2007), } \\
\text { IBGE (2007) }\end{array}$ \\
\hline$I T_{t-1}$ & $\begin{array}{c}\text { Telecomunicaçõ } \\
\text { es por } \\
\text { trabalhador } \\
\end{array}$ & + & $\begin{array}{l}\text { Zhang e Fan } \\
\qquad(2004)\end{array}$ & $\begin{array}{c}\text { Ferreira e } \\
\text { Malliagros } \\
(1998)\end{array}$ & $\begin{array}{c}\text { ANATEL (2007), } \\
\text { MTE (2007) }\end{array}$ \\
\hline Kagro $_{t-1}$ & $\begin{array}{c}\text { Capital } \\
\text { Agropecuário } \\
\text { por trabalhador }\end{array}$ & + & Solow (1956) & $\begin{array}{c}\text { Bonelli e Pessoa } \\
\text { (1998) }\end{array}$ & $\begin{array}{l}\text { IBGE (2007), } \\
\text { MTE (2007). }\end{array}$ \\
\hline$P \& D_{t-1}$ & $\begin{array}{c}\text { Número } \\
\text { pesquisadores } \\
\text { da Embrapa por } \\
\text { trabalhador } \\
\end{array}$ & + & $\begin{array}{c}\text { Evenson, } \\
\text { Waggoner e } \\
\text { Ruttan (1979) }\end{array}$ & $\begin{array}{c}\text { Bonelli e Pessoa } \\
\text { (1998) }\end{array}$ & $\begin{array}{c}\text { EMBRAPA } \\
\text { (2007), MTE } \\
(2007) .\end{array}$ \\
\hline$C O N A B_{t-1}$ & $\begin{array}{l}\text { Capacidade de } \\
\text { armazenagem }\end{array}$ & + & $\begin{array}{l}\text { Nogueira Jr.; } \\
\text { Tsunechiro } \\
(2005)\end{array}$ & $\begin{array}{l}\text { Zhang e Fan } \\
\qquad(2004)\end{array}$ & CONAB (2007) \\
\hline$P \& D_{t-1}^{*} \ln \left(Y_{t-1}\right)$ & $\begin{array}{l}\text { Interação entre } \\
\text { logaritmo } \\
\text { natural do PIB } \\
\text { por trabalhador } \\
\text { agropecuário e } \\
\text { número de } \\
\text { pesquisadores }\end{array}$ & - & $\begin{array}{c}\text { Barro e } \\
\text { Sala-i-Martin } \\
\quad(1995)\end{array}$ & $\cdots$ & $\begin{array}{l}\text { IBGE (2007); } \\
\text { MTE (2007); } \\
\text { EMBRAPA } \\
\quad(2007)\end{array}$ \\
\hline Cred $_{t-1}$ & $\begin{array}{c}\text { Crédito rural } \\
\text { por trabalhador }\end{array}$ & + & $\begin{array}{c}\text { Gasques e Villa } \\
\text { Verde (2003). }\end{array}$ & $\cdots$ & $\begin{array}{c}\text { BACEN (2007); } \\
\text { MTE (2007). }\end{array}$ \\
\hline Irri $_{t-1}$ & Área irrigada & + & $\begin{array}{l}\text { Zhang e Fan } \\
\qquad(2004)\end{array}$ & $\begin{array}{c}\text { Zhang e Fan } \\
\qquad(2004)\end{array}$ & MENDES (2005) \\
\hline
\end{tabular}

Fonte: Elaboração própria.

\footnotetext{
${ }^{12}$ Obs.: Todos os valores serão deflacionados utilizando-se o deflator implícito do PIB para o ano de 2000.
} 
5. A área total irrigada ( Irri $\left._{t-1}\right)$ foi obtida nos censos agropecuários do Brasil (IBGE), em publicações especializadas e interpolada pela taxa de crescimento do período. A área total plantada foi obtida nos censos agropecuários do Brasil, em 1985 e 1995 (IBGE, 2005), e interpoladas e extrapoladas com a taxa geométrica de crescimento do período. A pesquisa utilizou a relação entre a área total irrigada e a plantada ${ }^{13}$.

6. Crédito rural por trabalhador $\left(C r e d_{t-1}\right)$ é o valor referente à agregação do saldo contábil das operações de crédito rural (inclui custeio, investimento e comercialização do ciclo produtivo), realizadas pelas agências das instituições financeiras dos municípios até 31 de dezembro de cada ano.

7. O número de pesquisadores por trabalhador $\left(P \& D_{t-1}\right)$ foi obtido na Embrapa ${ }^{14}$.

8. Variável de interação entre o logaritmo natural do PIB por trabalhador inicial e o número de pesquisadores da Embrapa por trabalhador $\left(P \& D_{t-1}^{*} \ln \left(Y_{t-1}\right)\right)$.

9. Estoque de capital estadual por trabalhador $\left(\right.$ Kagrot $\left._{t-1}\right)$ foi deflacionado pelo IPC (ano-base 2000) em função da participação estadual na utilização total da terra segundo as unidades da federação. Essa proxy foi obtida do estoque de capital do setor agropecuário brasileiro (Kagrot-1BR), ponderado pela participação estadual na área total plantada.

Nesse caso, tem-se:

$$
\text { Kagro }_{t-1}=\text { Kagro }_{t-1 B R} * \frac{\text { áreaplantada }}{\text { estado }}
$$

É importante destacar que as variáveis descritas anteriormente também serão consideradas defasadas espacialmente, de forma que se avaliem como os seus aspectos de transbordamento ou externalidades espaciais são significativos. No tocante aos coeficientes das variáveis representadas por $P \& D_{t-1}$, cabe informar que os mesmos permitem avaliar se pesquisa contribuiu para o crescimento do PIB agropecuário por trabalhador dos estados. Por sua vez, também é avaliado o efeito de interação, $P \& D_{t-1}{ }^{*} \ln \left(Y_{t-1}\right)$, para verificar se a pesquisa agropecuária contribuiu na diminuição das desigualdades regionais do PIB agropecuário por trabalhador. ${ }^{15}$

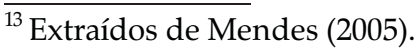

${ }^{14}$ Ver anexo.

${ }^{15}$ A inserção das variáveis interativas é baseada em Barro e Sala-i-Martin (1995, p. 432), que utilizaram a interação entre o PIB e o capital humano em um estudo empírico para verificar uma possível difusão tecnológica.
} 


\section{Resultados e discussão}

Esta seção é reservada à análise econométrica dos coeficientes estimados, da qualidade de ajuste das regressões e do diagnóstico. Vale destacar que o modelo teve 509 observações ${ }^{16}$, durante o período de 19 anos e com os 26 estados mais o Distrito Federal.

Tabela 1. Resultados da estimação por MQVD para a variável taxa de crescimento do PIB por trabalhador agropecuário, nos estados do Brasil, 1986 a 2004.

\begin{tabular}{lrrrc}
\hline Variáveis & Coeficiente & Desvio padrão & Estatística-t & Probabilidade \\
\hline Kagro $_{t-1}$ & 0.000452 & 0.003741 & 0.120806 & 0.9039 \\
$P \& D_{t-1}$ & 22.69186 & 6.992026 & 3.245391 & 0.0013 \\
CONAB $B_{t-1}$ & -0.037125 & 0.255675 & -0.145205 & 0.8846 \\
$I T_{t-1}$ & -0.003663 & 0.001197 & -3.058697 & 0.0023 \\
$C r e d_{t-1}$ & $2.62 \mathrm{E}-07$ & $4.23 \mathrm{E}-07$ & 0.619713 & 0.5357 \\
$D R_{t-1}$ & 4.604084 & 3.242609 & 1.419870 & 0.1563 \\
Irrit-1 $^{*} \ln \left(Y_{t-1}\right)$ & $-1.23 \mathrm{E}-07$ & $5.05 \mathrm{E}-07$ & -0.244126 & 0.8072 \\
$\left.P \& D_{t-1} \ln \right)$ & -4.235526 & 1.171978 & -3.613998 & 0.0003 \\
$\ln \left(Y_{t-1}\right.$ & -0.205021 & 0.037629 & -5.448444 & 0.0000 \\
$R^{2}$ & 0.209671 & & & \\
$R^{2}$ ajustado & 0.151191 & & & 0.0000 \\
Estatística F & 3.585294 & & & 0.0000 \\
Teste de Hausman & 55.36527 & & & 0.0000 \\
White & 7.3447 & & & 0.0000 \\
Jarque-Bera & 9.365741 & & & 0.0000 \\
Breusch-Pagan-Godfrey & 53.4367 & & & 0.6572 \\
Durbin-Wu-Hausman & 1.295 & & & \\
\hline
\end{tabular}

Fonte: Resultados da pesquisas.

O modelo de efeitos fixos sem dependência espacial foi estimado por mínimos quadrados com variáveis dummies (MQVD). De acordo com a Tabela 1, os diagnósticos indicam que os erros não são normais. Pelo teste de White e pelo teste de Breusch-Pagan-Godfrey (BPG), há evidências de erros heterocedásticos. As observações discrepantes podem invalidar a suposição clássica de normalidade dos resíduos, bem como a heterocedasticidade pode implicar uma matriz de variância-covariância sem a diagonal principal constante. Nesses casos, as estimações por MQO podem ser muito inconsistentes e ineficientes.

Em relação à heterocedasticidade, todas as próximas regressões foram estimadas, utilizando-se os desvios padrões robustos baseados na matriz de

\footnotetext{
${ }^{16}$ Deve-se levar em consideração a ausência de informações para o estado de Tocantins antes de 1988.
} 
variância-covâriancia de White com o intuito de eliminá-la. Pelo teste de Hausman, conclui-se que o procedimento mais adequado é o modelo de efeitos fixos. $\mathrm{O}$ teste Durbin-Wu-Hausman indica que a variável $P \& D_{t-1}$ é exógena em relação ao modelo.

A Tabela 2 apresenta os resíduos do modelo estimado para cada ano do período de 1986 a 2004. Observa-se que a dependência espacial está presente em quase todos os anos. Essa evidência é confirmada pelos valores do índice I de Moran computado nos resíduos durante cada ano, pois se observa que a hipótese nula de nenhuma dependência espacial nos resíduos é rejeitada. Assim, fica clara a necessidade de se incorporar a correção espacial no modelo.

Existem sinais da importância da dimensão espacial na dinâmica do crescimento econômico, pois, uma vez identificada a dependência espacial no crescimento do PIB por trabalhador agropecuário dos estados brasileiros, qualquer modelo econométrico estimado que utilize o crescimento do PIB por trabalhador agropecuário como variável dependente deve considerar esta dependência.

Tabela 2. Índice I de Moran para os resíduos dos dados de painel de efeitos fixos estimados pelo modelo MQO, em cada período de tempo (1986 a 2004) ${ }^{17}$.

\begin{tabular}{ccccrc}
\hline Ano & I de Moran & Média & D-padrão & \multicolumn{1}{c}{$\mathbf{Z}$} & P-value \\
\hline 1986 & -0.1951368 & -0.038 & 0.039422 & -3.892545 & 0.0038 \\
1987 & 0.2354248 & -0.038 & 0.039422 & 8.444526 & 0.0000 \\
1988 & 0.2395451 & -0.038 & 0.039422 & 8.825877 & 0.0000 \\
1989 & 0.336547 & -0.038 & 0.039422 & 9.757354 & 0.0000 \\
1990 & 0.2739463 & -0.038 & 0.039422 & 7.036544 & 0.0000 \\
1991 & 0.1625873 & -0.038 & 0.039422 & 6.052541 & 0.0000 \\
1992 & 0.06525713 & -0.038 & 0.039563 & 3.636547 & 0.0085 \\
1993 & -0.1399543 & -0.038 & 0.039458 & -2.300021 & 0.0188 \\
$\mathbf{1 9 9 4}$ & $\mathbf{- 0 . 0 6 3 6 5 4 9}$ & $\mathbf{- 0 . 0 3 8}$ & $\mathbf{0 . 0 3 8 9 6 7}$ & $\mathbf{- 0 . 7 2 0 2 1 4 0}$ & $\mathbf{0 . 4 6 7 3}$ \\
1995 & 0.09335741 & -0.038 & 0.039419 & 4.000241 & 0.0008 \\
1996 & 0.07136854 & -0.038 & 0.039428 & 2.780050 & 0.0053 \\
$\mathbf{1 9 9 7}$ & $-\mathbf{0 . 0 1 3 6 5 7 4 4}$ & $\mathbf{- 0 . 0 3 8}$ & $\mathbf{0 . 0 3 8 5 8 2}$ & $\mathbf{0 . 5 4 0 8 0 4}$ & $\mathbf{0 . 5 8 3 6}$ \\
1998 & 0.2998524 & -0.038 & 0.038440 & 8.1802413 & 0.0000 \\
1999 & 0.33654217 & -0.038 & 0.038903 & 10.04000 & 0.0000 \\
2000 & 0.33657413 & -0.038 & 0.038593 & 9.901147 & 0.0000 \\
2001 & 0.3636528 & -0.038 & 0.038862 & 10.4228409 & 0.0000 \\
2002 & 0.46936541 & -0.038 & 0.039671 & 11.806354 & 0.0000 \\
2003 & 0.2735287 & -0.038 & 0.039226 & 7.930214 & 0.0000 \\
2004 & -0.1368577 & -0.038 & 0.039727 & -2.2102417 & 0.0264 \\
\hline
\end{tabular}

Fonte: Resultados da pesquisas.

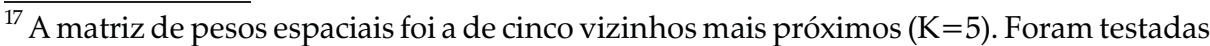
as matrizes de pesos espaciais de $k$-vizinhos mais próximos de $k=4$ a $k=10$, e as matrizes Rook e Queen (com $1^{\circ}, 2^{\circ}$ e $3^{\circ}$ ordens de vizinhança). Contudo, foi utilizada a matriz que apresentou maior valor da estatística I de Moran em todo o período da análise, seguindo o procedimento proposto por Baumont (2004).
} 
Os resultados das estimativas encontram-se também na Tabela $3 .{ }^{18}$ Em termos de qualidade de ajuste, o melhor modelo, avaliado com base nos critérios de Informação de Akaike (AIC) e Schwarz (SC), é o modelo de efeitos fixos com defasagem espacial.

Ressalta-se que o modelo de defasagem espacial é o mais adequado, dado que o coeficiente caracteriza-se o efeito da vizinhança sobre a dinâmica de crescimento foi negativo e significativo. Em relação ao fato da defasagem espacial $\left(W_{1} G_{t}\right)$ apresentar significância estatística, pode-se afirmar que a evolução do crescimento econômico da renda agropecuária por trabalhador dos estados de determinada região pode ser influenciada pela evolução do crescimento de rendo dos estados vizinhos. Por outro lado, o fato de a defasagem espacial possuir sinal negativo indica que a interação espacial da taxa de crescimentos da renda agropecuária por trabalhador entre os estados brasileiros tem um padrão de dispersão espacial, predominando, em média, estados com taxas de crescimento elevadas vizinhos de estados com taxas baixas (padrão alto-baixo) e vice versa (padrão baixo-alto).

Tabela 3. Resultados das regressões do modelo espacial de dados de painel para a variável taxa de crescimento do PIB agropecuário por trabalhador (1986 a 2004) ${ }^{\mathbf{1 9}}$.

\begin{tabular}{lcccc}
\hline Variável & Coeficiente & Desvio padrão & Estatística-t & Probabilidade \\
\hline Kagrot-1 $_{t-1}$ & 0.029063 & 0.025388 & 1.144736 & 0.2529 \\
$P \& D_{t-1}$ & $\mathbf{5 8 6 . 3 1 9 4}$ & $\mathbf{1 3 8 . 8 9 4 1}$ & $\mathbf{4 . 2 2 1 3 4 3}$ & $\mathbf{0 . 0 0 0 0}$ \\
CONAB $_{t-1}$ & -2.188174 & 1.672240 & -1.308528 & 0.1913 \\
$D R_{t-1}$ & -1.621319 & 1.958201 & -0.827963 & 0.4081 \\
$I T_{t-1}$ & 0.029114 & 0.628382 & 0.046332 & 0.9631 \\
Cred & $\mathbf{7 . 1 9 E - 0 6}$ & $\mathbf{2 . 6 8 E - 0 6}$ & $\mathbf{2 . 6 8 1 0 8 5}$ & $\mathbf{0 . 0 0 7 6}$ \\
P\& $_{t-1}{ }^{*} \ln \left(Y_{t-1}\right)$ & $\mathbf{- 9 5 . 1 3 8 7 0}$ & $\mathbf{2 3 . 1 7 7 0 5}$ & $\mathbf{- 4 . 1 0 4 8 6 7}$ & $\mathbf{0 . 0 0 0 0}$ \\
$W_{1} G_{t}$ & $\mathbf{- 0 . 3 9 4 2 4 1}$ & $\mathbf{0 . 1 2 7 0 0 4}$ & $\mathbf{- 3 . 1 0 4 1 6 3}$ & $\mathbf{0 . 0 0 2 0}$ \\
$\ln \left(Y_{t-1}\right)$ & $\mathbf{- 0 . 4 1 2 3 1 7}$ & $\mathbf{0 . 1 0 4 0 0 3}$ & $\mathbf{- 3 . 9 6 4 4 8 4}$ & $\mathbf{0 . 0 0 0 1}$ \\
Irrit $_{t-1}$ & $6.55 \mathrm{E}-07$ & $3.70 \mathrm{E}-07$ & 1.768830 & 0.0776 \\
\hline Estatística F & 6.109387 & & & \\
Critério de Akaike & 0.605378 & & & \\
Critério de Schwarz & 0.931357 & & & \\
\hline
\end{tabular}

Fonte: Resultados da pesquisas.

\footnotetext{
${ }^{18}$ Foram estimados modelos de defasagem espacial, erro espacial, bem como outros modelos espaciais. O modelo escolhido, contudo, foi definido pelos critérios de decisão de Akaike e Schwarz, indicando o modelo de defasagem espacial como o mais adequado.

${ }^{19}$ As variáveis de controle defasadas espacialmente não-significativas foram excluídas do modelo.
} 
Na Tabela 3, percebe-se que o coeficiente da variável $\ln \left(Y_{t-1}\right)$ é significativamente diferente de zero e de sinal negativo, confirmando, com isso, a hipótese de convergência agropecuária de renda por trabalhador entre os estados brasileiros, ou seja, a desigualdade entre os estados está diminuindo no tempo.

Segundo Chatterji (1992), para garantir que a variável em estudo sofra a convergência do período inicial até o final, é necessário que $-2<\beta<0$. Em outros termos, a tabela mostra que existe convergência do tipo condicional para o crescimento do PIB por trabalhador agropecuário para os estados brasileiros. Esses resultados são mais confiáveis porque se utilizam outras variáveis para condicionar o modelo.

Entre os coeficientes reportados na Tabela 3, merece destaque o coeficiente da variável interativa que visa controlar interativamente a influência do nível de renda inicial e da medida de pesquisa e desenvolvimento, que se mostrou significativo e negativo. Isso pode ser interpretado como sendo evidências de que os investimentos de pesquisa contribuem efetivamente para a convergência estadual da renda agropecuária por trabalhador.

Já os efeitos de $P \& D_{t-1}$ sobre o crescimento foram positivos. O coeficiente para a proxy para pesquisa e desenvolvimento agropecuário por trabalhador $\left(P \& D_{t-1}\right)$ foi estatisticamente significativo e de sinal positivo, com uma alta magnitude. Esse resultado oferece evidências em favor da hipótese de McCunn e Huffman (2000) de que a pesquisa agropecuária regional gera externalidades espaciais, elevando a produtividade.

Outro resultado importante foi de que a estimativa do coeficiente para crédito rural $\left(\mathrm{Cred}_{t-1}\right)$ para o crescimento do PIB por trabalhador agropecuário foi confirmada no modelo. Gasques et al. (2004) ${ }^{20}$ apontam que no período de 1975 a 2002 o aumento da produção do agronegócio se dá quase que exclusivamente apoiado no crescimento da produtividade e que os gastos com pesquisa são mais importantes que o crédito rural na explicação da produtividade total dos fatores da agropecuária. De modo geral, esses resultados, apontados em Gasques et al. (2004), são corroborados aqui, pois, de forma semelhante, a pesquisa agropecuária e o crédito rural são as variáveis relevantes para explicar o crescimento da renda agropecuária neste artigo.

${ }^{20}$ No período de 1975 a 2002, os autores analisaram os fatores condicionantes da PTF da agropecuária. Utilizando como variáveis explicativas: gastos da Embrapa (proxy de pesquisa em agropecuária); desembolsos do crédito rural para produtores e cooperativas, incluindo créditos do Pronaf; e relação entre os preços recebidos pelos agricultores e os pagos pelos insumos adquiridos (relação de trocas). 


\section{Considerações finais}

Este trabalho constatou que pesquisa em agropecuária é relevante para o crescimento da renda agropecuária bem como para a convergência da renda agropecuária entre os estados brasileiros. Os resultados econométricos também sugeriram que a pesquisa agropecuária é determinante na atividade produtiva e redistributiva, quando se considera todos os estados do Brasil. Nesse sentido, descontinuidades nos recursos para o sistema de pesquisas agropecuárias terão impactos sobre a geração e a difusão de inovações para o setor e, por conseqüência, influências na expansão e convergência da renda agropecuária.

Ao discutir o caráter espacial da distribuição do crescimento do PIB por trabalhador agropecuário por meio dos estados do Brasil, este trabalho visa a contribuir para o redimensionamento da questão regional rural no Brasil.

Os resultados obtidos mostraram a existência de convergência condicional no período estudado. No modelo econométrico de convergência condicional, o crescimento do PIB por trabalhador agropecuário foi afetado por variáveis como crédito rural e externalidades espaciais. Merece destaque a confirmação da hipótese de que os investimentos em pesquisa agropecuária influenciaram positivamente o crescimento e contribuíram para convergência de renda agropecuária brasileira.

Em termos de políticas públicas, estas deveriam ter como alvo o incentivo de isenções tributárias para investimentos em pesquisa agropecuária e financiamento de capital de risco para empresas intensivas em tecnologia agropecuária mais avançada.

Uma importante implicação para o planejamento de pesquisas regionais, extraído do presente artigo, é o fato de que investimentos públicos em pesquisas agropecuárias devem ser feitos de forma regional, corroborando a hipótese de McCunn e Huffman (2000). Isto porque o efeito de transbordamento é maior quando os investimentos são feitos de forma regional. Em outros termos, a instalação de um centro de pesquisas em um determinado local causa um efeito de transbordamento tecnológico entre as regiões próximas ao instituto, proporcionando maiores ganhos de escala no que diz respeito ao aumento da produtividade e à melhora de outros fatores de produção agrícolas.

Trabalhos posteriores poderão estender a análise de convergência no setor agropecuário para um outro conjunto de variáveis. A inclusão de uma variável que medisse o grau de capacitação do pessoal ocupado no campo como e/ou inserção de pesquisadores de órgãos estaduais bem como dos centros universitários. 


\section{Referências Bibliográficas}

AGÊNCIA NACIONAL DE ENERGIA ELÉTRICA - ANEEL. Disponível em: <http://www.aneel.gov.br/fiscalizacao>. Acesso em: 10 jun. 2007.

AGÊNCIA NACIONAL DE TELECOMUNICAÇÕES - ANATEL. Disponível em: <http://www.anatel.gov.br>. Acesso em: 10 jun. 2007.

ALMEIDA, E. S.; PEROBELLI, F. S.; FERREIRA, P. G. C.. Existe convergência espacial da produtividade agrícola no Brasil? XI Encontro Regional de Economia/Nordeste: Estratégias de Desenvolvimento Regional, BNB/ANPEC. Fortaleza-CE. 19, 20 e 21 de julho de 2006. p.21

ANSELIN, L. Spatial Econometrics: Methods and Models. Studies in Operational Regional Science, Kluwer Academic Publishers, Dordrecht, 284p. 1988

ARBIA, G.. Spatial econometrics: statistical foundations and applications to regional convergence. Berlin: Springer, 2006, 208 p.

ARELLANO, M. e BOND, S.. Some testes of specification for panel data: Monte Carlo evidence and an application to employment equations". Review of Economics Studies, v. 58, 277-297, 1991.

BANCO CENTRAL DO BRASIL - BANCEN. Disponível em: < www.bcb.gov.br/?CREDRURAL>. Acesso em: 15 nov. 2007.

BARRETO, R. C. S.. Desenvolvimento regional e convergência de renda nos municípios do Ceará. (tese de doutorado da UFV), Viçosa/MG, f. 191, 2007.

BARRO, R.J.; SALA-I-MARTIN, X.. Economic Growth. McGraw-Hill, 539p., 1995.

BARRO, R.J.; SALA-I-MARTIN, X. Convergence across states and regions. Brookings Papers on Economic Activity, New-Haven, Connecticut, USA, v. 1, p. 107-182, 1991.

BAUMONT, C. Spatial effects in housing price models: do house prices capitalize urban development policies in the agglomeration of Dijon (1999)? Mimeo., Université de Bourgogne, 2004. Disponível em http://ungaro.u-bourgogne.fr/ baumont/e2004-04.pdf\#search $=\% 22 \% 22 \mathrm{k} \%$ 20nearest $\% 20$ neighbors $\% 22 \% 20$ likeli hood $\% 20 \% 22$ spatial $\% 20$ econometrics $\% 22 \% 22$. (acesso em 11/09/2006).

BEINTEMA, N.M.; AVILA, A.F.D.; PARDEY, P.G.. PED Agropecuário: Política, Investimentos e Desenvolvimento Institucional. Washington, D.C.: IFPRI, EMBRAPA, and Fontagro, agosto 2001. p116

BONELLI, R. ; PESSOA, E. P.. O papel do estado na pesquisa agrícola no Brasil. Rio de Janeiro: IPEA, 1998. 40p. (Texto para Discussão ,576). 
CHATTERJI M. Convergence clubs and endogenous growth. Oxford Review of Economic Policy, 8, p. 57-69, 1992.

COMPANHIA NACIONAL DE ABASTECIMENTO - CONAB. Disponível em: <http://www.conab.gov.br/armazenagem>. Acesso em: 15 nov. 2007.

DEPARTAMENTO NACIONAL DE INFRA-ESTRUTURA TERRESTRE - DNIT. Disponível em: <http://www.dnit.gov.br/pnv>. Acesso em: 20 jul. 2007.

ELHORST, P.J.. Specification and Estimation of Spatial Panel Data Models, International Regional Sciences Review, 26, 3:244-268. 2003.

EMPRESA BRASILEIRA DE PESQUISA AGROPECUÁRIA - EMBRAPA. Disponível em: <http://www.embrapa.gov.br>. Acesso em: 15 nov. 2007.

EVENSON, R. E., WAGGONER, P. E., RUTTAN, V.W.. Economic benefits from research: an exemple from agriculture. Science, v. 205, Sept., 1979.

GASQUES, J.G.; VILLA VERDE, C.M.. Gastos públicos na agricultura: evolução e mudanças. Rio de Janeiro: IPEA, 2003. 31 p. (Texto para discussão, 948).

GASQUES, J.G.; REZENDE, G.C.; VERDE, C.M.V.; SALERMO, M.S.; CONCEIÇÃO, J.C.P.R.; CARVALHO, J.C.S. Desempenho e crescimento do agronegócio no Brasil: textos para discussão 1009. IPEADATA, Brasília, 2004.40p.

GUTIERREZ L. . Convergence in US and EU Agriculture. European Review of Agricultural Economics, 27, 2,p. 187-206, 2000.

HOWITT, P.; MAYER-FOULKES, D.. R\&D, Implementation, and Stagnation: A Schumpeterian Theory of Convergence Clubs, Journal of Money, Credit and Banking, 37 (February 2005): 147-77.

INSTITUTO BRASILEIRO DE GEOGRAFIA E ESTATÍSTICA - IBGE. Disponível em: <http://www.ibge.gov.br/pam>. Acesso em: 15 dez. 2007.

INSTITUTO DE PESQUISA ECONÔMICA APLICADA - IPEA. Séries históricas -2007. Disponível em: < http://www.ipeadata.gov.br>. Acesso em: 10 dez. 2007.

JOHNSTON, J.; DINARDO, J.. Econometric methods. New York, $4^{\mathrm{o}}$ Ed., McGraw-Hill, 1997.

LUCAS, R. On the mechanics of economic development. Journal of Monetary Economics, v. 22, n. 1, p. 3-42, jul. 1988.

LUSIGI, A., PIESSE, J. AND THIRTLE, C.G.. Convergence of Por trabalhador Incomes and Agricultural Productivity in Africa. Journal of International Development, 10 (1), 1998, 105-115. 
MCCUNN, A.; HUFFMAN, W, E. Convergence in U.S Productivity Growth for Agriculture: Implications of Interstate Research Spillovers for Funding Agricultural Research. American Agricultural Economics Association, 2000.

MENDES, S. M.. Relação entre os Investimentos em Infra-Estrutura e a Produtividade Total dos Fatores na Agricultura Brasileira, 1985-2004.(tese de mestrado da UFV), Viçosa/MG, f. 111, 2005 .

Ministério do Trabalho e Emprego (MTE). Disponível em: $<$ http://www.mte.gov.br/caged >. Acesso em: 25 nov. 2007.

NOGUEIRA JÚNIOR, S.; TSUNECHIRO, A. Produção agrícola e infra-estrutura de armazenagem no Brasil. Informações Econômicas, v. 35, n. 2, p. 7-18, 2005.

OLIVEIRA JÚNIOR, J. N. ; CASTELAR, I. ; FERREIRA, R. T. . Convergência Microregional no Setor Agrícola Usando um Modelo Threshold. In: XXXV Encontro Nacional de Economia, 2007, Recife. Anais do XXXV Encontro Nacional de Economia, 2007.p.19

ROMER, P. M.. Increasing returns and long-run growth. Journal of Political Economy, Chicago, v. 94, n. 5, p. 1002-1037, Oct. 1986.

SOLOW, R. M.. A contribution to the theory of economic growth. Quarterly Journal of Economics, v. 70, n. 1, p. 65-94, Feb. 1956. Disponível em: $<$ www.wren-network.net/resources/2005-11.solow.contribution.pdf $>$.

SUHARIYANTO, K., THIRTLE, C.. Asian agricultural productivity and convergence. Journal of Agricultural Economics , vol. 52(3), 96- 110. 2001.

ZHANG, X.; FAN, S. How productive is infrastructure? A new approach and evidence from rural India. American Journal of Agricultural Economics, v. 86, n. 2, p. 492-501, 2004. 


\section{Apêndice}

Organização da Embrapa

A Embrapa está organizada em 13 unidades centrais, localizadas em sua sede, em Brasília, e 41 unidades descentralizadas, distribuídas em todo o território brasileiro. A empresa possui atualmente 8.230 empregados, dos quais 2.207 pesquisadores, sendo $27 \%$ com mestrado e $72 \%$ com doutorado.

A Embrapa não tem centros de pesquisa em cinco dos 26 estados brasileiros, quais sejam: Alagoas, Maranhão, Mato Grosso, Rio Grande do Norte e Tocantins. A distribuição das unidades da Embrapa se dá por principais produtos em cada estado: mandioca, frutas tropicais, cacau; recursos naturais, seringueira (Bahia); algodão (Paraíba); hortaliças, pós-colheita, recursos genéticos, biotecnologia, cerrados, bovinos, frutas, grãos (Distrito Federal); agrobiologia, solos, ecologia, meio-ambiente, tecnologia de alimentos (Rio de Janeiro); arroz de sequeiro, feijão (Goiás); agroindústria tropical, caju, frutas, plantas ornamentais, flores, ovinos e caprinos, pastagens (Ceará); instrumentação, biotecnologia, agroindústria, monitoramento e avaliação ambiental , informática agropecuária, gado de corte, gado de leite, pastagens , agroecologia , recursos naturais (São Paulo); floresta, soja , girassol (Paraná); carne, gado de corte, pastagem, recursos naturais, grãos, pecuária, pesca, meio-ambiente (Mato Grosso do Sul); gado de leite, milho, sorgo, biotecnologia, café (Minas Gerais); suínos, aves (Santa Catarina); trigo, cevada, centeio, aveia, soja , biotecnologia, uva, vinho, frutas temperadas, solos, arroz irrigado, gado de leite, recursos naturais, gado de corte, pastagens (Rio Grande do Sul); agrofloresta (Amazonas); agrofloresta (Acre); agrofloresta (Amapá); agrofloresta, pastagens (Rondônia); agrofloresta (Roraima); irrigação, frutas, grãos (Piauí); produção animal, solos, coco, cacau, frutas , hortaliças (Sergipe); recursos naturais, irrigação, produção animal, frutas (Pernambuco); agrofloresta, recursos naturais, meio-ambiente, agroindústria (Pará); café, frutas, gado de leite, recursos naturais (Espírito santo). 


\section{Anexo 1}

Distribuição das informações estatísticas utilizadas para o ano de 2004, por Unidades da Federação (UF)

\begin{tabular}{|c|c|c|c|c|c|c|c|c|c|}
\hline طبّد & 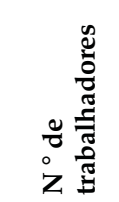 & 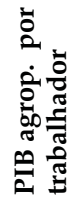 & 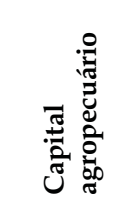 & 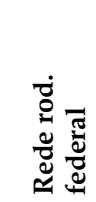 & 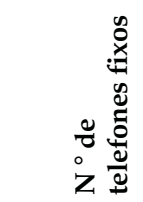 & 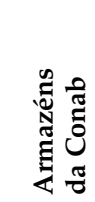 & : & 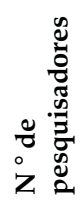 & 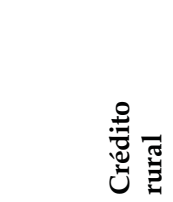 \\
\hline $\mathrm{AC}$ & 3.770 & 33 & 12.401 & 1.234 & 98.889 & 28 & 730 & 36 & 51.790 .869 \\
\hline $\mathrm{AL}$ & 19.792 & 35 & 11.954 & 963 & 316.752 & 280 & 75.080 & 12 & 76.429 .628 \\
\hline $\mathrm{AM}$ & 9.385 & 118 & 12.156 & 1.752 & 447.405 & 45 & 1.920 & 74 & 32.035 .814 \\
\hline $\mathrm{AP}$ & 846 & 169 & 2.611 & 680 & 80.423 & 1 & 2.070 & 22 & 2.295 .636 \\
\hline BA & 84.937 & 90 & 153.957 & 11.917 & 2.118 .845 & 2.848 & 292.330 & 212 & 580.453 .340 \\
\hline CE & 29.381 & 48 & 45.417 & 3.573 & 974.072 & 383 & 76.140 & 127 & 130.770 .761 \\
\hline $\mathrm{DF}$ & 6.698 & 40 & 1.194 & 473 & 884.950 & 306 & 12.010 & 313 & 66.416 .318 \\
\hline ES & 35.926 & 29 & 19.258 & 1.600 & 813.202 & 880 & 98.750 & 19 & 204.488 .482 \\
\hline GO & 65.869 & 103 & 155.360 & 6.588 & 1.451 .963 & 11.068 & 197.700 & 174 & 1.697.257.079 \\
\hline MA & 12.354 & 161 & & 4.612 & & & & 35 & 198.329.007 \\
\hline MG & 269.658 & 39 & 224.111 & 18.215 & 4.236 .951 & 6.216 & 350.200 & 322 & 2.196.301.316 \\
\hline MS & 56.916 & 86 & 187.729 & 4.867 & 538.836 & 5.798 & 89.970 & 206 & 1.093 .902 .921 \\
\hline MT & 80.511 & 64 & 389.503 & 6.880 & 651.393 & 14.900 & 18.530 & 90 & 1.941.282.356 \\
\hline PA & 64.388 & 99 & 126.207 & 1.783 & 717.368 & 271 & 7.480 & 229 & 320.588 .883 \\
\hline РB & & 82 & 21.492 & 1.727 & 432.192 & 64 & 48.600 & 77 & 101.803 .463 \\
\hline PE & 65.076 & 53 & 28.861 & 2.935 & 1.235 .266 & 643 & 98.480 & 121 & 86.245 .819 \\
\hline PI & 6.434 & 85 & 49.079 & 4.871 & 300.463 & 174 & 2.6780 & 65 & 95.794 .701 \\
\hline PR & 182.109 & 71 & 93.264 & 6.692 & 2.577 .095 & 20.375 & 72.240 & 364 & 3.850 .399 .481 \\
\hline RJ & 49.741 & 20 & 11.237 & 2.458 & 5.028 .552 & 222 & 39.330 & 161 & 49.068 .782 \\
\hline $\mathrm{RN}$ & 24.325 & 31 & 19.701 & 1.863 & 398.444 & 66 & 18.220 & 13 & 91.348 .960 \\
\hline $\mathrm{RO}$ & 18.155 & 66 & 76.835 & 1.357 & 255.921 & 343 & 4.920 & 46 & 242.263.189 \\
\hline RR & 930 & 62 & 24.326 & 1.606 & 65.961 & 18 & 9.210 & 27 & 10.527.331 \\
\hline RS & 173.508 & 88 & 122.713 & 9.365 & 2.729 .350 & 20.140 & 1.086 .000 & 396 & 4.092 .460 .138 \\
\hline SC & 125.656 & 58 & 36.346 & 3.495 & 1.595 .100 & 3.371 & 143.420 & 169 & 1.736 .468 .198 \\
\hline SE & 9.314 & 66 & 9.322 & 349 & 280.307 & 18 & 48.970 & 89 & 49.710 .365 \\
\hline SP & 503.431 & 67 & 92.247 & 6.169 & 1.3473 .304 & 9.668 & 499.800 & 751 & 3.567 .822 .745 \\
\hline $\mathrm{TO}$ & 9.807 & 49 & 99.082 & 2.458 & 165.527 & 1.352 & 73.350 & 8 & 286.573.149 \\
\hline
\end{tabular}

Fonte: Dados da pesquisa.

Nota: as informações de fluxo de credito rural são referentes ao ano de 2003. 
Anexo 2

Variação percentual (\%) das informações estatísticas utilizadas no período de 1985-2003, por Unidades da Federação (UF)

\begin{tabular}{|c|c|c|c|c|c|c|c|c|c|}
\hline "بْد: & 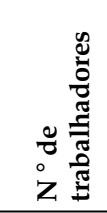 & 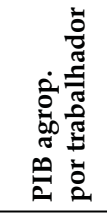 & 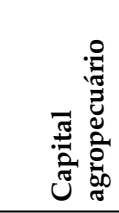 & 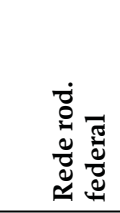 & 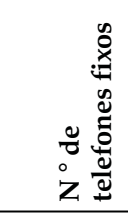 & 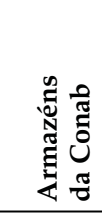 & 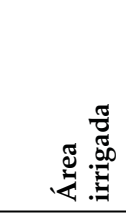 & 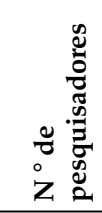 & 胥 \\
\hline $\mathrm{AC}$ & 257,69 & $-85,05$ & $-64,23$ & 513,93 & 869,79 & 13,60 & $1.303,85$ & 111,76 & 65,56 \\
\hline $\mathrm{AL}$ & 101,00 & $-73,69$ & $-23,64$ & 41,41 & 741,69 & $-59,05$ & 169,94 & $-52,00$ & $-83,60$ \\
\hline AM & $-21,89$ & 42,27 & $-68,67$ & 105,15 & 581,69 & $-60,18$ & 573,68 & $-10,84$ & $-48,72$ \\
\hline $\mathrm{AP}$ & $-30,14$ & 147,34 & $-67,36$ & 729,27 & 917,50 & $-60,00$ & $7.292,86$ & 175,00 & $-8,20$ \\
\hline BA & 226,24 & $-68,43$ & $-30,46$ & 213,28 & 726,72 & 214,78 & 173,07 & 10,42 & $-61,83$ \\
\hline CE & 92,86 & $-65,69$ & $-37,71$ & 99,83 & 569,68 & $-40,40$ & 13,13 & 115,25 & $-78,82$ \\
\hline $\mathrm{DF}$ & 54,90 & 90,18 & $-42,52$ & 190,18 & 367,32 & 134,20 & 116,87 & 73,89 & 39,48 \\
\hline ES & 116,49 & $-81,61$ & $-25,35$ & 124,40 & 675,01 & 87,34 & 98,31 & $-62,00$ & $-59,07$ \\
\hline GO & 559,28 & $-62,58$ & $-21,45$ & 98,43 & 987,16 & 192,36 & 887,76 & 65,71 & $-22,86$ \\
\hline MA & 90,85 & $-34,07$ & $-56,11$ & 155,23 & 952,78 & 70,17 & 100,72 & 16,67 & $-32,93$ \\
\hline MG & 212,60 & $-74,22$ & $-26,17$ & 119,80 & 500,69 & 98,92 & 79,94 & $-9,80$ & $-10,39$ \\
\hline MS & 407,63 & $-64,40$ & $-8,88$ & 116,02 & 646,61 & 161,53 & 248,61 & 53,73 & $-15,11$ \\
\hline MT & 524,26 & $-22,61$ & 55,45 & 248,18 & $1.259,11$ & 644,29 & 56,28 & 350,00 & 35,56 \\
\hline PA & 193,14 & $-29,92$ & $-22,93$ & 179,91 & 706,86 & 3,31 & $-37,23$ & 49,67 & 39,83 \\
\hline PB & 313,78 & $-72,50$ & $-33,39$ & 62,31 & 599,05 & $-66,84$ & 157,21 & 32,76 & $-66,04$ \\
\hline PE & 175,36 & $-52,38$ & $-34,95$ & 24,73 & 761,08 & 67,55 & 18,00 & 11,01 & $-87,33$ \\
\hline PI & 167,75 & $-61,55$ & $-37,34$ & 143,31 & 575,82 & 27,30 & 97,49 & 71,05 & $-50,60$ \\
\hline PR & 84,71 & $-36,90$ & $-15,67$ & 135,88 & 441,12 & 33,91 & 129,50 & 31,88 & $-27,26$ \\
\hline RJ & $-35,21$ & 23,35 & $-48,02$ & 62,89 & 345,36 & $-68,97$ & $-44,61$ & 10,27 & $-73,46$ \\
\hline $\mathrm{RN}$ & 386,31 & $-71,99$ & $-32,13$ & 73,63 & 815,65 & $-67,62$ & 3,59 & 30,00 & $-53,26$ \\
\hline RO & 319,96 & $-55,99$ & 92,32 & 90,32 & 948,47 & 376,81 & $3.340,56$ & 4,55 & 246,87 \\
\hline RR & 360,40 & $-80,79$ & 70,89 & $3.915,00$ & 706,47 & 13,13 & 311,16 & 350,00 & 59,79 \\
\hline RS & 66,55 & $-7,33$ & $-22,22$ & 90,85 & 576,18 & 40,69 & 39,31 & 37,50 & $-31,43$ \\
\hline SC & 64,84 & $-4,59$ & $-26,03$ & 84,04 & 864,97 & 60,00 & 88,83 & 40,83 & 20,81 \\
\hline SE & 46,49 & $-26,21$ & $-26,63$ & 8,05 & 695,02 & $-63,20$ & 587,68 & 12,66 & $-74,62$ \\
\hline SP & 26,23 & 68,20 & $-31,20$ & 429,98 & 389,64 & $-16,36$ & $1.658,99$ & 121,53 & $-26,90$ \\
\hline TO & 674,03 & $-84,13$ & $5.395,73$ & 215,13 & $1.303,25$ & 100,12 & 94,28 & 0,00 & $-70,21$ \\
\hline
\end{tabular}

Fonte: Dados da pesquisa.

Nota: As informações iniciais de Tocantins são de 1989. 